\title{
Pitch Discrimination Learning: Specificity for Pitch and Harmonic Resolvability, and Electrophysiological Correlates
}

\author{
Samuele Carcagno ${ }^{1,2}$ and Christopher J. Plack ${ }^{3}$ \\ ${ }^{1}$ Department of Psychology, Lancaster University, Lancaster, LA1 4YF, UK \\ ${ }^{2}$ CNRS and Université de Bordeaux, BP 63, 146 Rue Leo Saignat, F-33076, Bordeaux, France \\ ${ }^{3}$ Division of Human Communication and Deafness, The University of Manchester, Manchester, M13 9PL, UK
}

Received: 29 November 2010; Accepted: 15 March 2011; Online publication: 12 April 2011

\begin{abstract}
Multiple-hour training on a pitch discrimination task dramatically decreases the threshold for detecting a pitch difference between two harmonic complexes. Here, we investigated the specificity of this perceptual learning with respect to the pitch and the resolvability of the trained harmonic complex, as well as its cortical electrophysiological correlates. We trained 24 participants for $12 \mathrm{~h}$ on a pitch discrimination task using one of four different harmonic complexes. The complexes differed in pitch and/or spectral resolvability of their components by the cochlea, but were filtered into the same spectral region. Cortical-evoked potentials and a behavioral measure of pitch discrimination were assessed before and after training for all the four complexes. The change in these measures was compared to that of two control groups: one trained on a level discrimination task and one without any training. The behavioral results showed that learning was partly specific to both pitch and resolvability. Training with a resolved-harmonic complex improved pitch discrimination for resolved complexes more than training with an unresolved complex. However, we did not find evidence that training with an unresolved complex leads to specific learning for unresolved complexes. Training affected the P2 component of the cortical-evoked potentials, as well as a later component (250-400 ms). No significant changes were found on the mismatch negativity (MMN) component, although a separate experiment showed that this measure was sensitive to pitch

Correspondence to: Samuele Carcagno - CNRS and Université de Bordeaux · BP 63, 146 Rue Leo Saignat, F-33076, Bordeaux, France. Telephone: +33-5-57571651; email: samuele.carcagno@u-bordeaux2.fr
\end{abstract}

changes equivalent to the pitch discriminability changes induced by training. This result suggests that pitch discrimination training affects processes not measured by the MMN, for example, processes higher in level or parallel to those involved in MMN generation.

Keywords: auditory training, pitch learning, P2, MMN, evoked potentials

\section{INTRODUCTION}

Pitch is a perceptual attribute of periodic sounds that plays a crucial role in the segregation and identification of auditory events (Plack and Oxenham 2005). For harmonic complex tones, sounds comprising multiple harmonically related frequency components, pitch generally corresponds to the fundamental frequency $\left(F_{0}\right)$. Even if the $F_{0}$ component is physically removed from a harmonic complex, a "residue pitch" corresponding to the $F_{0}$ is heard (Licklider 1956). The frequency resolution of the cochlea progressively decreases with increasing center frequency (Glasberg and Moore 1990), but the partials of harmonic complexes usually have a constant spacing corresponding to the $F_{0}$. As a result, low-numbered harmonics tend to fall into different auditory filters and are said to be "resolved," while higher numbered harmonics cannot be separated out into different frequency channels and are said to be "unresolved." The residue pitch is perceived whether the harmonics of a complex are resolved into different auditory filters or interact with each other within auditory filters. However, the pitch is 
less salient in the second case (Houtsma and Smurzynski 1990; Plack and Carlyon 1995). While the $F_{0}$ of resolved harmonics can be computed on the bases of either a rate-place code or a temporal code, the $F_{0}$ of unresolved harmonics can be coded only temporally (de Cheveigné 2005). This has led to the proposition that two different mechanisms may be used to encode the $F_{0}$ of resolved- and unresolved-harmonic complexes (Houtsma and Smurzynski 1990; Carlyon and Shackleton 1994).

In support of this dual pitch mechanism theory, Grimault et al. (2002) found that $F_{0}$ discrimination learning with harmonic complexes was partly specific to the resolvability of their partials. Listeners trained with a resolved-harmonic complex showed greater improvements with other untrained resolved-harmonic complexes than with unresolved-harmonic complexes, and vice versa. This result suggests that $F_{0}$ discrimination training affects pitch processing mechanisms that are specific to resolved and unresolved harmonics. On the other hand, studies on $F_{0}$ discrimination learning have not found evidence that this learning is pitch-specific (see Wright and Zhang 2009 for a review). Pitch specificity, however, has been tested only for stimuli that, while having the same pitch, were presented in different frequency regions. We sought to revisit the effects of resolvability and pitch on $F_{0}$ discrimination learning controlling for this possible confound. This was achieved by including a condition in which unresolved harmonics were combined in alternating phase (Shackleton and Carlyon 1994) to produce a complex with the same pitch and the same spectral region as a comparison resolved-harmonic complex.

Improvements in pure tone frequency discrimination have been associated with changes in the P2, a sensory component of the cortical-evoked potentials (EPs), and the mismatch negativity $(\mathrm{MMN})$, a later component of the EPs elicited by the presentation of a rarely occurring sound in the context of a repeating sound (Atienza et al. 2002; Bosnyak et al. 2004). Another objective of this study was to examine the electrophysiological correlates of $F_{0}$ discrimination learning for missing fundamental harmonic complexes and determine whether or not they show the same pattern of specificity with respect to resolvability and pitch that is seen behaviorally. This may allow us to understand at what level of processing (P2, MMN) pitch-specific or resolvability-specific learning occurs.

To these ends, we trained naive listeners on an $F_{0}$ discrimination task with one of four harmonic complex tones. The complexes were all filtered into the same frequency region, but differed in resolvability and/or pitch. Before and after training, $F_{0}$ difference limens and EPs were recorded in response to all four complexes and compared to those of two control groups: one trained on a level discrimination task and one without any training. The control group trained on a level discrimination task allowed us to assess whether the training effects were specific to $F_{0}$ discrimination training or were more generic effects of auditory training. We also ran an additional MMN control experiment to measure the sensitivity of the MMN response to pitch changes equivalent to the pitch discriminability changes induced by training.

\section{METHODS}

\section{Participants}

Thirty-six participants (13 females, 34 right-handed, 2 ambidextrous) completed the experiment. The participants ranged in age between 19 and 33 years (mean $=24, \mathrm{SD}=2.9)$. They all had normal hearing for both ears with absolute pure tone thresholds below $20 \mathrm{~dB} \mathrm{HL}$ at octave frequencies from 250 to $8,000 \mathrm{~Hz}$. None had prior experience in psychoacoustic tasks or musical training. All participants gave written informed consent and were paid an hourly wage for their participation in the experiment. All procedures of the study were approved by the Department of Psychology Ethics Committee, Lancaster University.

\section{Stimuli}

The stimuli consisted of four harmonic complexes and a narrowband noise (NBN). The waveforms and spectra of all the stimuli are shown in Figure 1. All stimuli had a duration of $100 \mathrm{~ms}$, including 10 -ms rise and fall raised-cosine ramps. The complexes were band-pass-filtered between 1.1 and $2.2 \mathrm{kHz}(3-\mathrm{dB}$ cutoffs; nominal slope, $80 \mathrm{~dB} /$ octave) and had an overall level of $80 \mathrm{~dB}$ SPL. The base $F_{0}$ 's of two of the complexes were $110 \mathrm{~Hz}$, and the other two had base $F_{0}$ 's of 220 and $246.94 \mathrm{~Hz}$, respectively. In order to keep the spectrum level of the harmonic complexes constant, when the $F_{0}$ differed from the base $F_{0}$ (comparison complex in the psychophysical task or deviant complex in the EP recordings), its intensity was adjusted by a factor of $F_{0 x} / F_{0 \text { base }}$, where $F_{0 x}$ is the required $F_{0}$ and $F_{0 \text { base }}$ is the base $F_{0}$. The harmonics of one complex $\left(110-\mathrm{Hz} F_{0}\right)$ were added in alternating sine $\left(0^{\circ}\right)$ and cosine $\left(90^{\circ}\right)$ phase (ALT), while the harmonics of the other complexes were added in sine phase (SINE). For convenience, the harmonic complexes will be referred to as 110 ALT, 110 SINE, 220 SINE, and 246.94 SINE, respectively. For the 110 ALT and 110 SINE complexes, harmonics numbered 10 to 20 fall within the pass band of the filter and can be considered unresolved. For the 220 SINE and 246.94 SINE complexes, harmonics numbered 5 to 10 and 5 to 9 , respectively, fall within the pass band of the filter 


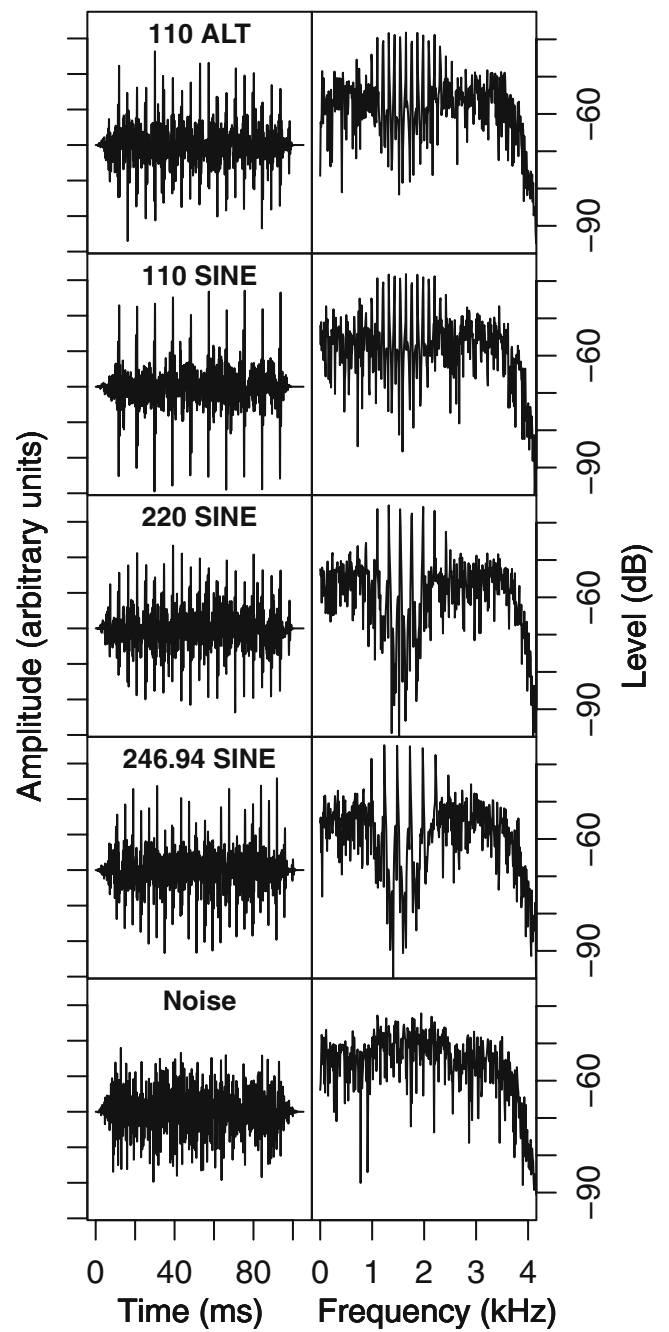

FIG. 1. Waveforms (left column) and spectra (right column) of the stimuli used in the experiment.

and can be considered mostly resolved (Shackleton and Carlyon 1994; Bernstein and Oxenham 2003). While the pitch of harmonic complexes generally corresponds to their $F_{0}$, unresolved-harmonic complexes summed in ALT phase have a pitch corresponding to double their $F_{0}$ (Shackleton and Carlyon 1994). Hence, the pitch of the 110 ALT complex is equal to the pitch of the 220 SINE complex. This was confirmed by the results of a short tone comparison task performed by all the listeners at the end of the main experiment. A lowpass noise with a $1.1-\mathrm{kHz}$ cutoff was added to all the complexes in order to mask possible combination tones. The spectrum level of the noise was $5 \mathrm{~dB}$ below the spectrum level of the complexes. Another band of noise with the same spectrum level was added in a higher frequency region between 2.4 and $3.4 \mathrm{kHz}$ to limit the available information to the region of the cochlea tuned to the frequency region of the complex.

The NBN was filtered between 1.1 and $2.2 \mathrm{kHz}(3-$ dB cutoffs; nominal slope, $80 \mathrm{~dB} /$ octave) and had an overall level of $80 \mathrm{~dB}$ SPL. The same two noise bands (low-pass $1.1 \mathrm{kHz}$, band-pass $2.4-3.4 \mathrm{kHz}$ ) were also added to the NBN stimulus at a spectrum level $5 \mathrm{~dB}$ below the spectrum level of the NBN. The NBN and the noise side bands for all stimuli were generated independently for each presentation during the psychophysical sessions, but were frozen samples for the electrophysiological sessions.

For the psychophysical sessions, the stimuli were generated digitally with 32-bit resolution and a $48-\mathrm{kHz}$ sampling rate on a Macintosh workstation. The stimuli were played through an M-AUDIO Firewire 410 24-bit digital-to-analog converter (DAC) and presented binaurally via Sennheiser HD580 headphones. For the electrophysiological sessions, all stimuli were generated digitally with $16-$ bit resolution and a $40-\mathrm{kHz}$ sampling rate. The stimuli were played through a DAC included in the EP data acquisition system (Intelligent Hearing Systems-Smart EP) and presented binaurally through mu-metal-shielded Etymotic ER2 insert earphones.

\section{Experimental Design}

All participants took part in a preliminary 1-h session (S0) during which they performed an audiometric test to ascertain normal hearing and two blocks of the discrimination task for each stimulus $\left(F_{0}\right.$ discrimination for the harmonic complexes, and level discrimination for the NBN) to familiarize themselves with the stimuli and procedures of the experiment. A timeline of the remaining sessions for the participants who took part in the training phase is shown in Figure 2. The second session (S-pre) lasted $1 \mathrm{~h}$ and $30 \mathrm{~min}$. In this session, participants performed first an additional practice block with each stimulus and then performed five blocks of the level discrimination task

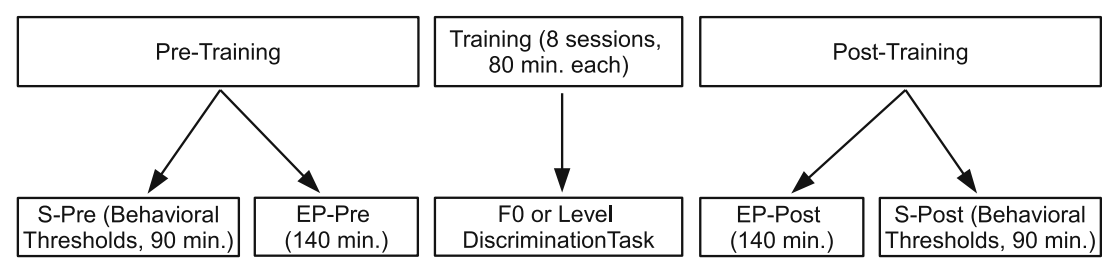

FIG. 2. Timeline of the experimental sessions for the participants who took part in the training phase. 
followed by five blocks of the $F_{0}$ discrimination task for each stimulus (in random order). The threshold estimates obtained in these last five blocks were used to measure baseline performance. Cortical EPs were recorded in a separate 2-h and 20-min session (EP-pre). After the baseline EP recording session, the first 30 participants were randomly assigned to one of the following groups to establish the stimulus they would be trained on: G110A, G110S, G220S, G246.94S, GLD. Participants of the first four groups were trained for eight sessions on an $F_{0}$ discrimination task, with one of the following stimuli: 110 ALT (G110A), 110 SINE (G110S), 220 SINE (G220S), 246.94 SINE (G246.94S). Participants of the GLD group were trained for eight sessions on a level discrimination task with the NBN. During the training sessions, participants had to complete a total of 28 blocks on the discrimination task. The duration of each training session was about $1 \mathrm{~h}$ and 20 min. The mean time between the session preceding the training phase (EP-pre) and the session following the training phase (EP-post) was 27 days $(\mathrm{SD}=9$ days). The remaining six participants were assigned to a no-training group (No-Tr) and waited for a period of time roughly equivalent to the mean time taken for the participants of the other groups to complete training (mean time between the EP-pre and the EP-post session was 31 days, $\mathrm{SD}=9$ days). After the training sessions, or equivalent amount of time for the No-Tr group, cortical EPs (EP-post session) were recorded in response to all stimuli. The final session (S-post) consisted of a second evaluation session of the performance on the discrimination tasks for all the stimuli. During this session, the participants first performed six blocks on the level discrimination task, followed by six blocks for each stimulus (in random order) on the $F_{0}$ discrimination task. At the end of the last session, all listeners performed two blocks of a tone comparison task aimed at testing whether the pitch of the 110 ALT complex would be perceived as more similar to the pitch of the 220 SINE or the 110 SINE complex.

\section{Psychophysical Tasks}

Listeners sat in a double-walled sound-attenuating booth in front of a computer monitor located outside the sound booth. $F_{0}$ and level difference limens (DLs) were measured with a three-interval, three-alternative forced-choice procedure with a two-down one-up adaptive rule tracking the $70.7 \%$ correct point on the psychometric function (Levitt 1971). On each trial, three observation intervals separated by $500 \mathrm{~ms}$ were presented, each containing a sound. For the $F_{0}$ discrimination task, two observation intervals contained a harmonic complex with a fixed $F_{0}$ (standard intervals), and one observation interval contained a harmonic complex of a lower $F_{0}$ which was varied adaptively (comparison interval). The order of the observation intervals was randomized for each trial. The $F_{0}$ of the harmonic complex in the standard intervals was 110 (ALT phase), 110 (SINE phase), 220, or $246.94 \mathrm{~Hz}$, depending on the stimulus being tested in that block. For the level discrimination task, two observation intervals contained an NBN with a spectrum level of $L \mathrm{~dB}$ SPL (standard intervals), while one observation interval contained an NBN with a spectrum level of $L-\Delta L \mathrm{~dB}$ SPL (comparison interval). The order of the observation intervals was randomized for each trial. The listener was asked to indicate by a key press on a numeric keypad which of the sounds was different from the other two (odd-one-out task). Feedback was always provided at the end of each trial through the presentation of a colored light on the computer screen. For the $F_{0}$ discrimination task, the percentage $F_{0}$ difference between the complexes in the standard and comparison observation intervals was initially set at $20 \%$ and was increased (after an incorrect response) and decreased (after two consecutive correct responses) by a factor of 2 for the first four turn points and by a factor of 1.414 thereafter. The maximum percentage $F_{0}$ difference allowed was $80 \%$. If the adaptive track called for an $F_{0}$ difference $>80 \%$, the $F_{0}$ difference was set at $80 \%$ and the track continued. For the level discrimination task, the level difference between the standard and the comparison intervals was initially set at $10 \mathrm{~dB}$ and was increased (after an incorrect response) and decreased (after two consecutive correct responses) by a factor of 2 for the first four turn points and by a factor of 1.414 thereafter. Sixteen turn points were measured for each block of trials and the $F_{0}$ or $\Delta L$ threshold estimate was taken as the geometric mean of the last 12 .

For the tone comparison task, participants were presented on each trial with three harmonic complexes-110 SINE, 110 ALT, and 220 SINE-separated by $500-\mathrm{ms}$ intervals. The order of presentation of the complexes was randomized between trials. The listener was asked to indicate by a key press on a numeric keypad which of the complexes sounded different from the other two. Each listener performed a total of 40 trials subdivided into two blocks. The aim of this task was to check that summing the harmonics of the $110-\mathrm{Hz} F_{0}$ complex in ALT phase would lead to a doubling of pitch compared to the case in which the harmonics were summed all in SINE phase. If the pitch of the 110 ALT complex is matched to $220 \mathrm{~Hz}$, listeners should choose the 110 SINE stimulus as the odd one in the comparison task. If the pitch of the 
110 ALT complex is instead matched to $110 \mathrm{~Hz}$, listeners should choose the 220 SINE stimulus as the odd one in the comparison task.

\section{EP Recordings}

Participants sat in a double-walled sound-attenuating room. The electroencephalogram (EEG) was recorded differentially between gold-plated scalp electrodes mounted on the frontal midline $(\mathrm{Fz})$, central midline $(\mathrm{Cz})$, and right mastoid $(\mathrm{RM})$ sites according to the 10-20 location system. A reference electrode was placed on the tip of the nose. Another electrode placed on the mid-forehead $(\mathrm{Fpz})$ served as the common ground. The inter-electrode impedances were maintained below $5 \mathrm{k} \Omega$. The EEG signal was recorded with a $1-\mathrm{kHz}$ sampling rate, amplified by a factor of 50,000, and band-pass filtered from 1 to $30 \mathrm{~Hz}$. Electrodes monitoring vertical eye movements were used in removing eye blink artifacts, as defined by epochs with voltage changes exceeding $64 \mu \mathrm{V}$. Subjects were instructed to ignore the auditory stimuli presented via earphones, to refrain from extraneous body movements, and to focus their attention exclusively on a self-selected movie played silently with subtitles on a 17-in. laptop situated in the booth. Stimuli were played with a repetition rate of 1.5 per second (567 ms ISI). The presentation of the stimuli followed an oddball paradigm, with rare $(p=0.14)$ deviant sounds presented in the context of frequent standard sounds. The deviant sounds had a lower $F_{0}$ (for harmonic complexes) or lower level (for the NBN) than the standards. For each stimulus, the $F_{0}$ or level difference between the deviant and the standard was equal to the threshold $F_{0}$ or level difference measured psychophysically for each participant during the S-pre session. The rationale behind this choice was that while the MMN response at threshold should be small, if the effects of training are reflected in the MMN response, any increase in detectability after training should result in a larger MMN (see Novitski et al. 2004 for effects of frequency deviance size on MMN amplitude). Setting the deviant at the pre-training threshold avoids possible ceiling effects for the pre-training recordings.

One hundred and thirty artifact-free responses to the deviants (acquired in two randomized blocks of 65) were recorded for each stimulus using the oddball paradigm. At the end of these recordings, 260 artifactfree responses (acquired in two randomized blocks of $130)$ to the deviants presented alone $(p=1)$ were recorded for each stimulus in order to derive the MMN as the difference wave between the response to the deviant sound presented in the oddball sequence and the same physical stimulus presented alone (identity MMN paradigm; Chandrasekaran et al.
2007). The overall duration of a session, including electrode placement, was about $2 \mathrm{~h}$ and $20 \mathrm{~min}$.

The EEG waveforms were band-pass-filtered off-line between 1 and $30 \mathrm{~Hz}$ with a zero phase shift digital filter, baseline corrected ( -150 to 0 ms pre-stimulus baseline), and re-referenced to the RM electrode. Grand averages were computed for each group, stimulus, and session separately. Difference waves were derived by subtracting the EP waveform obtained by presenting the deviant stimulus in the oddball paradigm (Dev-Odd) from the EP waveform obtained by presenting the same stimulus alone (Dev-Rep). The resultant identity MMN eliminates any acoustical difference between comparison stimuli. For each stimulus, the MMN peak was defined as the most negative value in the grand average difference wave for the Fz channel between 150 and $300 \mathrm{~ms}$. The MMN mean amplitude was computed as the mean amplitude of a 50-ms window centered at the MMN peak. The statistical analyses on the MMN mean amplitude were performed on the data from the $\mathrm{Fz}$ channel, where the MMN amplitude is expected to be maximal. P2 amplitudes were computed for the standard stimulus (Stn) presented in the oddball paradigm and the Dev-Rep stimulus. The P2 peak latencies were defined as the most positive value in the grand average waveform recorded at the Fz electrode between 150 and 250 ms (Bosnyak et al. 2004). The mean P2 amplitude was computed for the electrode at $\mathrm{Fz}$ as the mean amplitude of a 50-ms window centered at the $\mathrm{P} 2$ peak.

Visual inspection of the EP-pre and EP-post grand average waveforms revealed that an additional component of the EPs might be sensitive to training effects. This component manifested itself in the form of a greater negativity in the EP-post waveforms compared to the EP-pre waveforms in a latency window from about 250 to $400 \mathrm{~ms}$. This component will be referred to as the "late EP" component. Its amplitude was measured as the mean amplitude of the EP recorded at the Fz electrode in a fixed window going from 250 to $400 \mathrm{~ms}$.

\section{Statistical Analyses}

The change in performance between the S-pre and Spost sessions in the behavioral discrimination tasks was estimated as $\mathrm{DL}_{\text {pre }} / \mathrm{DL}_{\text {post }}$, where $\mathrm{DL}_{\text {pre }}$ and $\mathrm{DL}_{\text {post }}$ are the thresholds obtained at the S-pre and S-post sessions, respectively. Since a given proportional change in threshold expressed in percent $F_{0}$ difference, or in level difference, corresponds to the same proportional change in $d^{\prime}$ (Buus and Florentine 1991; Plack and Carlyon 1995), this measure should also provide an estimate of the change in detectability of $F_{0}$ or level differences after training. The statistical tests involving the psychophysical data were run on the log-transformed $\mathrm{DL}_{\text {pre }} / \mathrm{DL}_{\text {post }}$ ratios. For the EP 
components evaluated (MMN, P2, late EP), the dependent measure was the change in amplitude of the component between the EP-post and EP-pre sessions. For each dependent measure that was assessed, a list of comparisons aimed at answering a number of specific questions about the effects of training were performed.

Before computing test statistics, means and standard deviations of each dependent measure were computed for each combination of stimulus per group. Data points falling beyond \pm 2 standard deviations of the group mean for a given stimulus were considered outliers. No outliers were detected for any of the dependent measures.

Since the change in $F_{0}$ DL between the S-pre and S-post sessions did not differ significantly between the GLD and the No-Tr groups $[t(10)=0.81$, $p=0.435]$, in order to increase statistical power, the two groups were collapsed together into a single control group when testing training effects for the complexes. Similarly, since changes in the level discrimination performance did not differ significantly between the groups trained on $F_{0}$ discrimination and the No-Tr group [ $t(28)=0.69, p=0.498]$, these groups were collapsed together into a single control group when testing training effects for the level discrimination task.

When a test involved a comparison between independent samples, the Fligner-Killeen test of the homogeneity of variances between two groups (Conover et al. 1981) was first performed. In the case of unequal variances between the two groups, the Welch-Satterthwaite approximation to the degrees of freedom (Satterthwaite 1946) was used. Since the expected direction of change for the dependent measures was known, all $t$ tests were run as one-tailed tests, except where explicitly stated. The comparisons that were run are described below. The groups and stimuli included for each comparison are detailed in Table 1. (a) Effectiveness of $F_{0}$ discrimination training: This test compared the dependent measure (change in behavioral thresholds, P2 amplitude, MMN amplitude, or late EP amplitude) averaged across all harmonic complexes, between listeners trained on $F_{0}$ discrimination and listeners in the control groups (GLD and No-Tr groups). (b) Effect of resolvability for resolved-harmonic complexes: This test compared the dependent measure, averaged across the resolved complexes, between listeners trained with a resolved complex and listeners trained with an unresolved complex. To avoid a potential bias related to the fact that $246.94 \mathrm{~Hz}$ was the trained pitch for listeners of one of the groups trained with resolved complexes (G246.94S), but not for any of the listeners in the groups trained with unresolved complexes, the 246.94 SINE stimulus was excluded from the analysis for listeners in the G246.94S group only. (c) Effect of resolvability for unresolved-harmonic complexes: Same as test $\mathrm{b}$, but on unresolved complexes. To avoid a potential bias related to the fact that $110 \mathrm{~Hz}$ was the trained pitch for listeners of one of the groups trained with unresolved complexes (G110S), but not

\section{TABLE 1}

List of contrasts

\begin{tabular}{lll}
\hline Contrast & Sample 1 & Sample 2 \\
\hline a & G110A (110A, 110S, 220S, 246.94S) & GLD (110A, 110S, 220S, 246.94S) \\
& G110S (110A, 110S, 220S, 246.94S) & No-TR (110A, 110S, 220S, 246.94S) \\
G220S (110A, 110S, 220S, 246.94S) & \\
G246.94S (110A, 110S, 220S, 246.94S) & G110A (220S, 246.94S) \\
b & G220S (220S, 246.94S) & G110S (220S, 246.94S) \\
c G246.94S (220S) & G220S (110A, 110S) \\
& G110A (110A, 110S) & G246.94S (110A, 110S) \\
d & G110S (110A) & G110A (110S, 246.94S) \\
& G110A (110A, 220S) & G110S (110A) \\
G110S (110S) $220 S(110 S, 246.94 S)$ \\
G220S (110A, 220S) & G246.94S (220S) \\
G246.94S (246.94S) & G110A (Noise) \\
& GLD (Noise) & G110S (Noise) \\
& & G220S (Noise) \\
& & G246.94S (Noise)
\end{tabular}

For each contrast, the groups included in each sample are listed. The stimuli included for each group are listed in parentheses 
for any of the listeners in the groups trained with resolved complexes, the 110 SINE stimulus was excluded from the analysis for the listeners in the G110S group only. (d) Pitch specificity: This test involved only the groups trained on $F_{0}$ discrimination, and compared the dependent measure between complexes with the same pitch as the trained one and complexes with a different pitch. To avoid a potential confound of resolvability, for the G110S and G246.94S groups, only the stimulus with the same resolvability as the trained complex was used as the different pitch stimulus. (e) Effectiveness of level discrimination training: This test compared the dependent measure for the trained stimulus in the level discrimination group to the dependent measure for the same stimulus in the other groups $\left(F_{0}\right.$ discrimination and No-Tr groups).

\section{MMN Control Experiment}

Seventeen participants (11 males, 17 right-handed) ranging in age between 19 and 36 years ( mean $=23$, $\mathrm{SD}=4$ ) completed the experiment. They all had normal hearing for both ears with absolute pure tone thresholds below $20 \mathrm{~dB}$ HL at octave frequencies from 250 to $8,000 \mathrm{~Hz}$. None had prior experience in psychoacoustic tasks or musical training. All participants were paid an hourly wage for their participation in the experiment. Two of the harmonic complexes used in the training experiment were selected: one resolved (246.94 SINE) and one unresolved (110 ALT). Only two out of the four complexes were used to allow data collection to be completed in a single session. For both complexes, the MMN was recorded in response to two deviants. The "small" deviant had a $\Delta F_{0}$ difference from the standard $\left(\Delta F_{0 \text { small }}\right)$ equal to the average $F_{0}$ DL for that stimulus measured from all participants during the S-pre session of the training experiment. The "large" deviant had a $\Delta F_{0}$ difference from the standard equal to $\Delta F_{\text {osmall }} \times\left(F_{0} \mathrm{DL}_{\text {pre }}\right)$ $\left.F_{0} \mathrm{DL}_{\text {post }}\right)$, where $F_{0} \mathrm{DL}_{\text {pre }} / F_{0} \mathrm{DL}_{\text {post }}$ is the average $F_{0}$ DL change measured in the training experiment for the complex (110 ALT or 246.94 SINE) in the group of listeners that trained with it. Assuming that a change in $F_{0}$ DL is proportional to a discriminability change (Plack and Carlyon 1995), the difference in discriminability between the small and large deviants for each stimulus should be equivalent to the average change in discriminability observed in the training experiment.

The equipment and the testing procedures used for the EP recordings, as well as the algorithm used to find MMN peaks and compute MMN amplitudes, were the same as for the training experiment. One hundred and thirty artifact-free responses (acquired in two randomized blocks of 65) were recorded for each deviant using the oddball paradigm. At the end of these recordings, 260 artifact-free responses (acquired in two randomized blocks of 130) to the deviants presented alone $(p=1)$ were recorded for each stimulus. The purpose of this experiment was to compare MMN changes in response to (1) stimulus discriminability changes induced by training or (2) discriminability changes introduced by presenting deviants of different magnitudes. Therefore, for both stimuli, the difference in amplitude between the MMNs elicited by the large and small deviants was first computed. This measure was then averaged across the two stimuli and compared to the difference in amplitude between the post- and pre-training MMNs recorded from the participants trained on $F_{0}$ discrimination. The MMN responses from the training experiment included only the responses to the stimulus with which each participant had trained. If the $\mathrm{MMN}$ is more sensitive to a change in discriminability produced by a change in the $F_{0}$ of the deviant than it is to the same change in discriminability produced by training, the MMN difference measure should be greater in the control experiment than in the training experiment. This was tested using a twosample, one-tailed $t$ test.

\section{RESULTS}

\section{Tone Comparison Task}

The results of the tone comparison task confirmed that listeners generally perceived the pitch of the 110 ALT complex as more similar to the pitch of the 220 SINE than the 110 SINE complex. Due to experimenter error, two participants were not tested on this task. The remaining 34 participants chose as the odd-one-out stimulus the 110 SINE complex on $67.3 \%$ of the trials, the 220 SINE complex on $18.2 \%$ of the trials, and the 110 ALT complex on $14.5 \%$ of the trials. A paired $t$ test comparing the arcsine-transformed proportion of trials in which the 110 SINE and 220 SINE complexes were chosen as the odd one out revealed a significant difference $[t$ $(33)=5.718, p<0.001$, two-tailed].

\section{Learning Curves}

The mean DLs for the $F_{0}$ and level discrimination task across all the sessions for each group are shown in Figure 3. The DL changes over the training sessions indicate that all the groups trained on an $F_{0}$ discrimination task, as well as the group trained on the level discrimination task, showed a protracted decrease in thresholds across sessions. For the groups trained on an $F_{0}$ discrimination task, this 


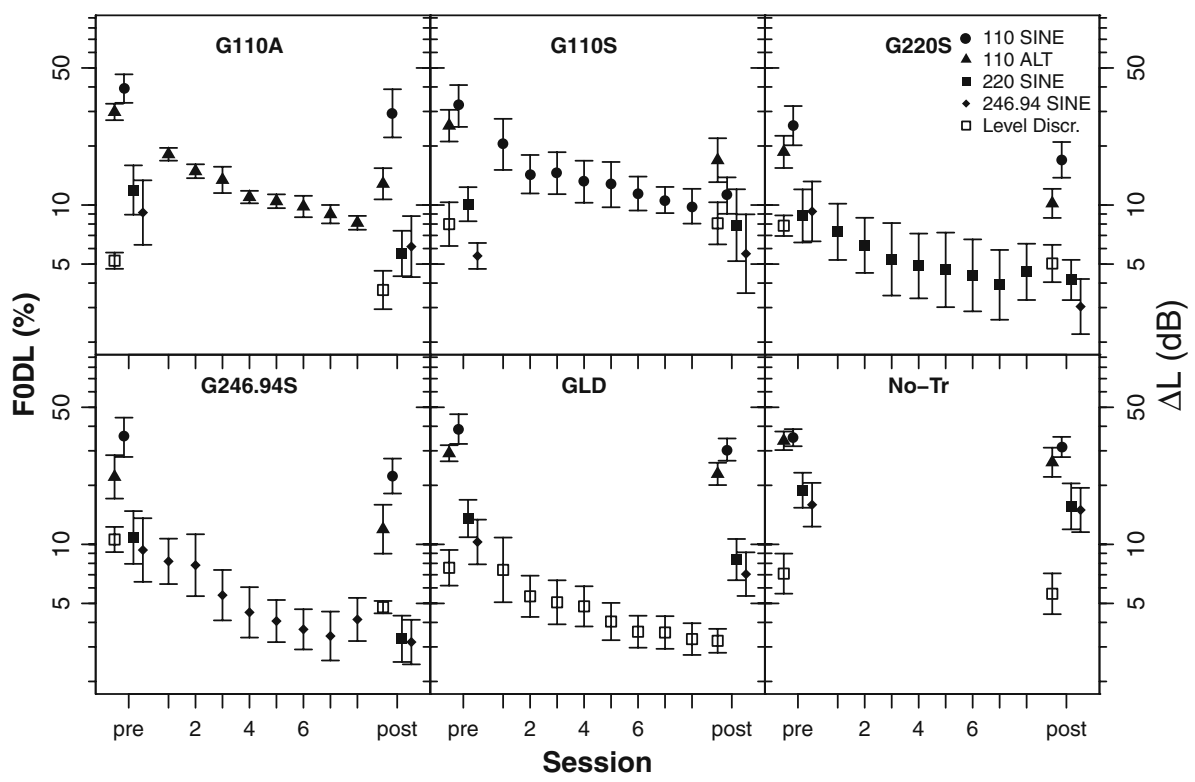

FIG. 3. Mean $F_{0}$ and $\Delta L \mathrm{DL}$ across sessions \pm 1 standard error. The filled symbols plot the $F_{0}$ DLs, the empty square plots the $\Delta L \mathrm{DL}$, and its scale should be referred to the axis on the right.

was confirmed by a repeated measures analysis of variance (ANOVA) on the log-transformed DLs with SESSION (1-8) as within-subjects and GROUP (G110S, G110A, G220S, and G246.94S) as betweensubjects factors. This analysis showed a significant effect of GROUP $[F(3,20)=4.24, p=0.018]$, which reflects the greater discriminability of the resolved compared to the unresolved-harmonic complexes (e.g., Shackleton and Carlyon 1994; Plack and Carlyon 1995). The effect of SESSION was also significant $[F(7,140)=24.07, p<0.001]$, while the GROUP $\times$ SESSION interaction was not significant $[F(21,140)=0.79, p=0.734]$. This indicates that a sustained learning curve was present irrespective of the resolvability status of the complex each group was trained with. The results of a univariate repeated measures ANOVA on the log-transformed DLs for the group trained on the level discrimination task also showed a significant effect of SESSION $[F(7,35)=9.26, p<0.001]$.

\section{Threshold Changes Between Pre- and Post-testing Sessions}

Figure 4 shows the changes in performance in the behavioral tasks between the S-pre and the S-post sessions. The set of planned contrasts for the DL change measure is shown in Figure 5, panel 1. The listeners trained on the $F_{0}$ discrimination task showed a significant decrease in threshold compared to the

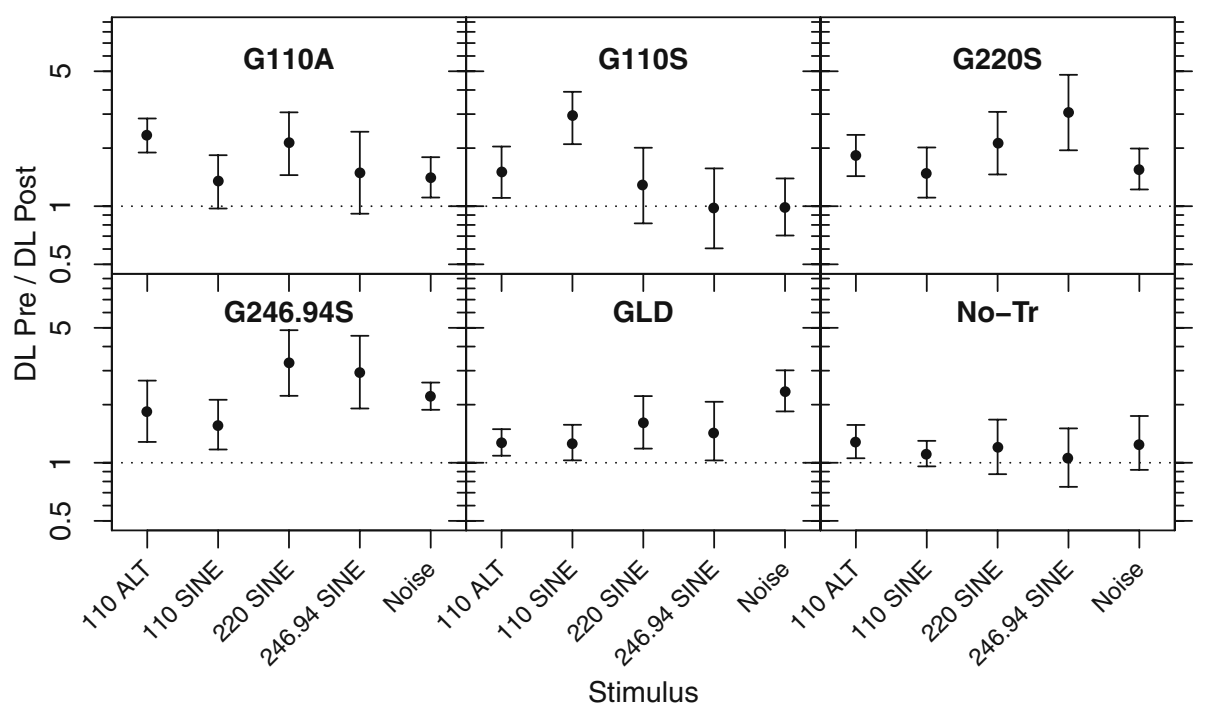

FIG. 4. Mean DL change between the S-Pre and S-Post sessions. Values above 1 (dotted line) indicate improvements. Error bars represent \pm 1 geometric standard error around the geometric mean ratio. 


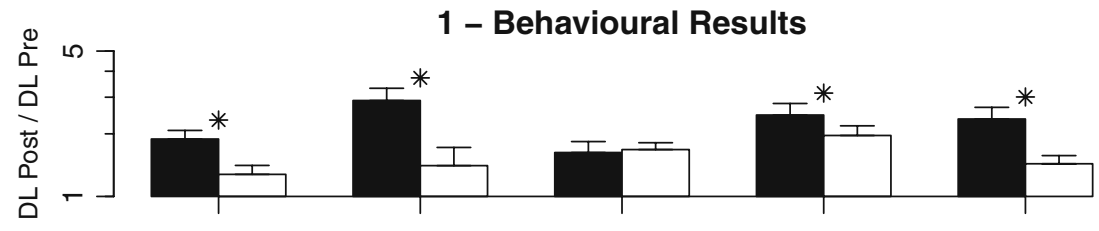

2-MMN
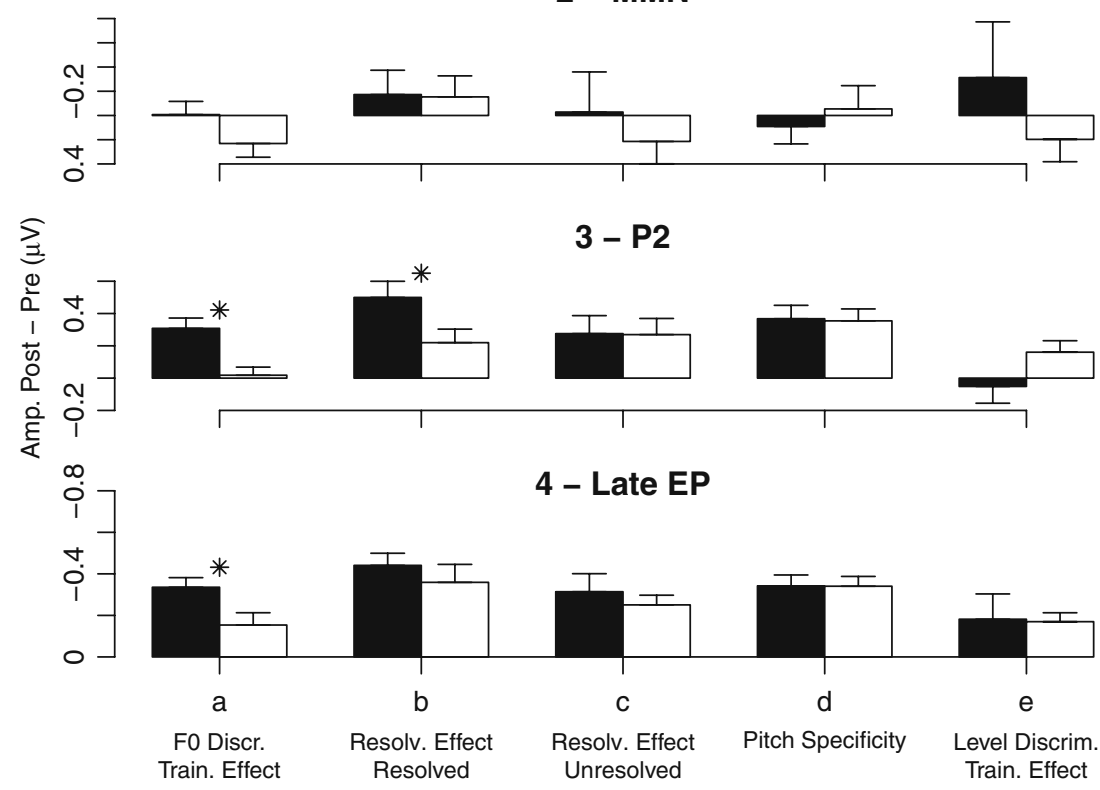

FIG. 5. Planned contrasts for all dependent measures. The samples used for each contrast are listed in Table 1. For each contrast, the filled bars represent sample 1 and the empty bars represent sample 2. Statistically significant contrasts are denoted by an asterisk. Error bars represent 1 standard error of the mean.

Contrast

control groups [comparison (a): $t(34)=2.57, p=0.007$ ] Listeners trained with a resolved complex improved significantly more than listeners trained with an unresolved complex on resolved complexes [comparison (b): $t(22)=2.97, p=0.004]$. However, the improvement for unresolved complexes was not significantly greater for listeners trained with an unresolved complex compared to listeners trained with a resolved one [comparison $(\mathrm{c}): t(18.47)=0.23, p=0.411]$. Thresholds decreased significantly more for stimuli with the trained than the untrained pitch [comparison (d): $t$ $(23)=2.05, p=0.026]$. Finally, the group trained on the level discrimination task showed a significant improvement on this task compared to the controls [comparison (e): $t(34)=2.34, p=0.013$ ].

\section{EP RESULTS}

The nose-referenced MMN is known to reach its maximum amplitude at $\mathrm{Fz}$, decrease at $\mathrm{Cz}$, and invert polarity at the mastoids (Novitski et al. 2004). Collapsing across stimuli, one-tailed $t$ tests revealed that the mean voltage of the nose-referenced MMN difference wave at session EPpre was significantly less than zero at the Fz electrode $[t$ $(35)=8.33, p<0.001]$, inverted polarity at the RM electrode $[t(35)=4.49, p<0.001]$, and was significantly more negative at the $\mathrm{Fz}$ than the $\mathrm{Cz}$ electrode $[t(35)=4.87, p<$
0.001]. This is consistent with the response recorded being the MMN. An MMN at session EP-pre was thus being elicited, even though the deviant stimuli were presented at the threshold level for each participant.

Figure 6 shows the mean MMN amplitude over the 50-ms window centered at the MMN peak at the EP-pre and EP-post sessions for all the groups. The set of planned contrasts for the MMN change measure is shown in Figure 5, panel 2. None of the comparisons was statistically significant for the MMN measure, suggesting that little effect of training was present on the MMN [comparison (a): $t(34)=1.37, p=0.09$; comparison (b): $t(22)=$ $0.07, p=0.471$; comparison (c): $t(22)=0.64, p=$ 0.264 ; comparison $(d): t(23)=0.63, p=0.266$; comparison (e): $t(34)=1.11, p=0.138]$.

Figure 7 shows the pre- and post-testing waveforms recorded from a representative participant in response to the standard stimulus presented in the oddball paradigm (Stn) for one of the complexes. ${ }^{1}$ The P2 can be seen peaking around $170 \mathrm{~ms}$. The mean P2 amplitude for the Stn and Dev-Rep stimuli averaged together is shown in Figure 8. The set of planned contrasts for the P2 change measure is shown in Figure 5, panel 3. Listeners trained on $F_{0}$ discrim-

\footnotetext{
1 The full set of the grand average EP plots may be obtained by contacting the first author.
} 


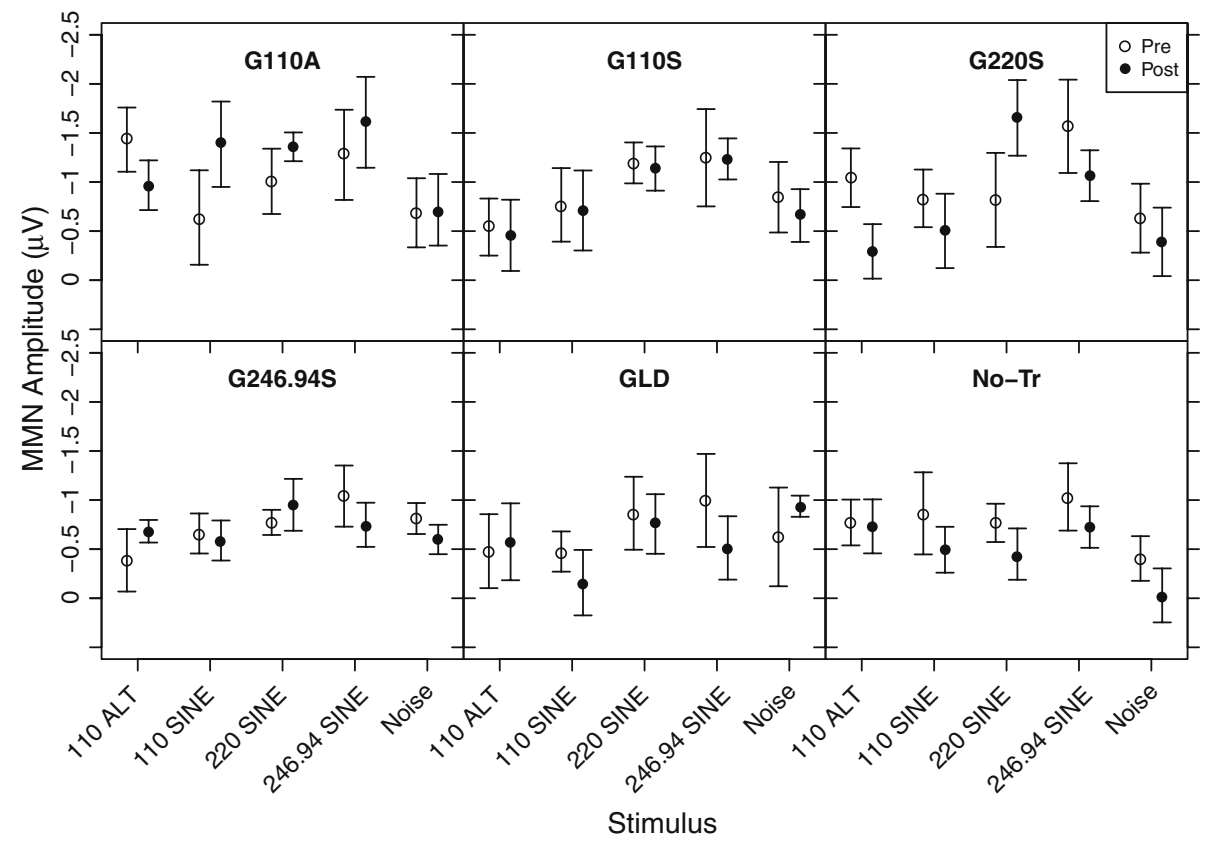

FIG. 6. Mean $M M N$ amplitude at the EP-pre and EP-post sessions. Error bars represent \pm 1 standard error of the mean.

ination showed a significant increase in P2 amplitude between the EP-pre and EP-post sessions for the pitchevoking stimuli compared to controls [comparison (a): $t(34)=2.98, p=0.003]$. For resolved complexes, this effect was significantly greater for listeners trained with a resolved complex than listeners trained with an unresolved complex [comparison (b): $t(22)=2.15, p=$ 0.022]. However, for the unresolved complexes, there was no significant difference between these two groups [comparison (c): $t(22)=0.04, p=0.484$ ]. The P2 did not show effects of pitch specificity [comparison $(d): t(23)=0.21, p=0.417]$. Finally, there was no significant P2 increase between the EP-pre and EPpost sessions for the narrowband noise in the level discrimination group compared to the other groups [comparison (e): $t(34)=1.27, p=0.106]$.

The late EP component can be observed as a greater post-training negativity between 250 and

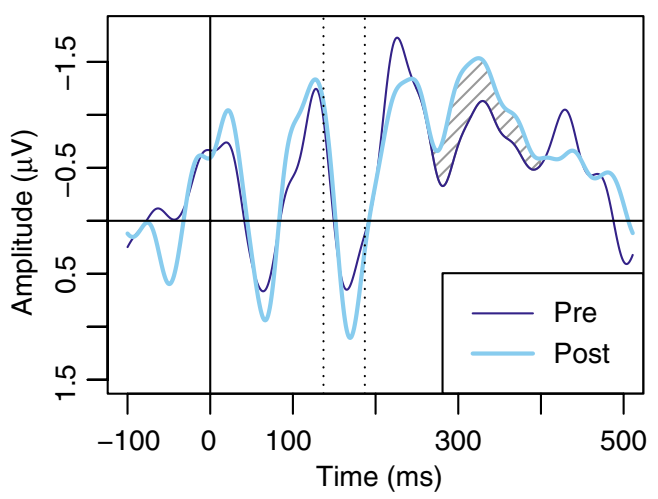

FIG. 7. EP traces from a representative participant in response to the Stn 220 SINE tone at the EP-pre (thin line) and EP-post (thick line) sessions. The P2 time window is enclosed by the vertical dotted lines. The shaded area indicates the time window over which the late EP component was measured.
$400 \mathrm{~ms}$ from the EP traces of a representative participant in Figure 7. Visual inspection of the nose-referenced grand average waveform indicates that this component reversed polarity at the mastoid electrode (voltages were more positive after training at the mastoid electrode). The mean amplitude of the late EP component for the Stn and Dev-Rep stimuli averaged together is shown in Figure 9. The list of contrasts for the late EP component is displayed in Figure 5, panel 4. All the contrasts for this component were based on two-tailed $t$ tests since an effect of training on this component was not expected, and thus the direction of change could not be predicted from previous studies. The amplitude of this component between the EP-pre and EP-post sessions became more negative for the pitch-evoking stimuli in the groups trained on $F_{0}$ discrimination compared to the control groups [comparison (a): $t(34)=2.34, p=$ $0.013]$. None of the contrasts related to resolvability [comparison (b): $t(22)=0.78, p=0.221$; comparison (c): $t(22)=0.64, p=0.264]$ or to pitch specificity [comparison (d): $t(23)=0.04, p=0.486]$ was significant. There was also no effect of level discrimination training on this component [comparison (e) : $t(34)=0.11, p=0.457]$.

\section{Correlations Between Behavioral and EP Changes}

We measured the correlations between the change in behavioral threshold and the change in amplitude of each EP component (P2, MMN, late EP) for each stimulus, collapsing across groups. For the P2 and MMN components, none of the correlations was statisti- 


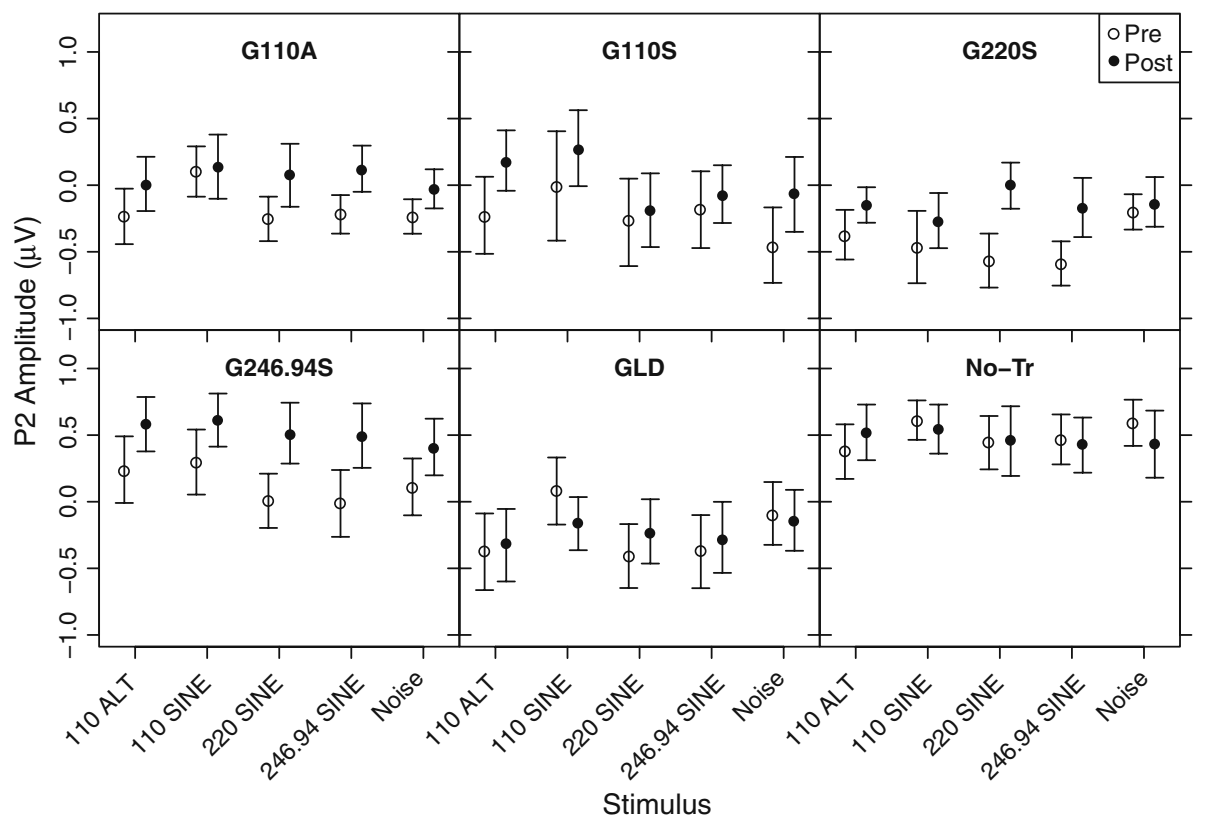

FIG. 8. Mean P2 amplitude for the Stn and Dev-Rep stimuli averaged together. Error bars represent \pm 1 standard error of the mean.

cally significant. For the late EP component, a significant negative correlation (greater post-training late EP negativity for larger threshold improvements) was present only for the $220 \mathrm{SINE}$ complex [ $r=-0.33, p=0.047]$. The negative correlation approached significance also for the other resolved complex 246.94 [ $r=-0.30, p=0.071]$.

\section{MMN Control Experiment}

Figure 10A shows the mean MMN amplitude over the 50-ms window centered at the MMN peak in response to the small and large deviants for each stimulus. The average MMN amplitude difference between large and small deviants across the two stimuli is shown in
Figure 10B, alongside the average MMN amplitude difference between the post- and pre-training MMN responses across all trained complexes of the training experiment. The $t$ test revealed that the MMN amplitude difference measure was significantly greater in the control experiment compared to the training experiment $[t(39)=1.84, p=0.037]$.

\section{DISCUSSION}

Behavioral Results

This experiment confirms earlier reports that $F_{0}$ discrimination training leads to a significant improve-

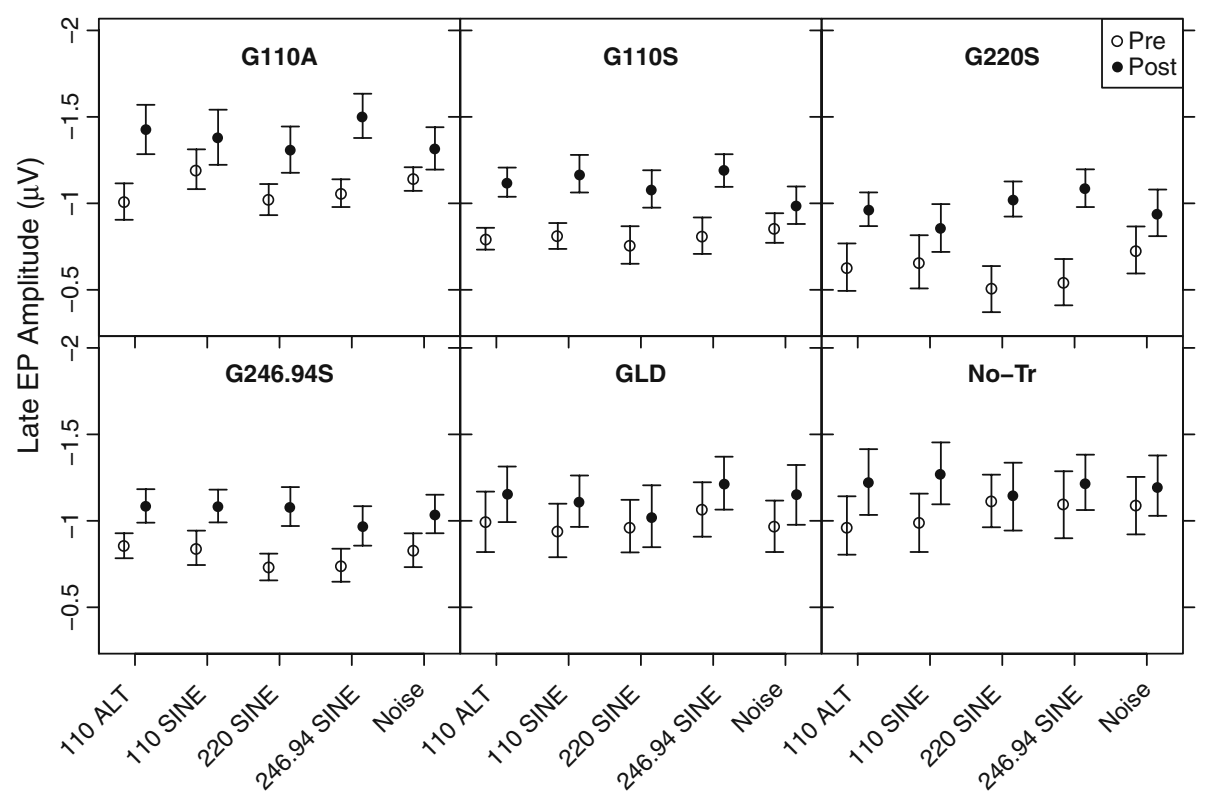

FIG. 9. Mean amplitude of the late EP component for the Stn and Dev-Rep stimuli averaged together. Error bars represent \pm 1 standard error of the mean. 

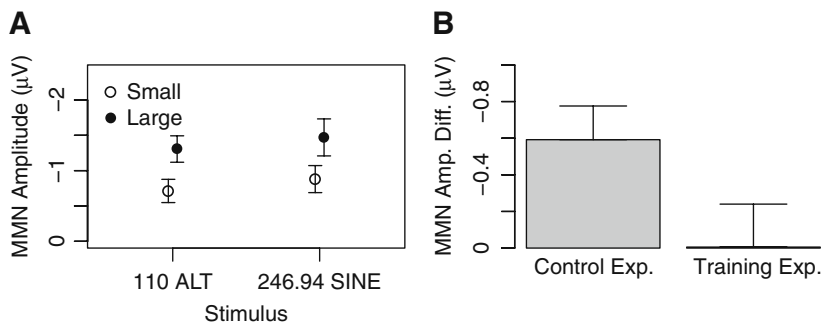

FIG. 10. A MMN amplitude for "large" and "small" deviants in the MMN control experiment. B MMN amplitude difference between "large" and "small" deviants for the MMN control experiment and between post- and pre-training deviants for the training experiment. Error bars represent \pm 1 standard error of the mean.

ment in pitch discrimination (Grimault et al. 2002; Demany and Semal 2002). Furthermore, the results of this experiment show that this improvement is partly pitch-specific for complexes filtered into the same frequency region. Pure tone frequency discrimination training is also partly pitch-specific: Although learning with one frequency transfers to untrained frequencies, this transfer is not complete (Wright and Zhang 2009). For $F_{0}$ discrimination learning, on the other hand, previous studies have not found evidence for pitch specificity. These studies assessed transfer of learning on the basis of pitch from a trained missing fundamental stimulus (e.g., harmonic complex or sinusoidally amplitude-modulated noise) either to a different stimulus type (e.g., pure tone, iterated rippled noise; Demany and Semal 2002; Grimault et al. 2003; Fitzgerald and Wright 2005) or to the same stimulus type filtered into a different frequency region (Grimault et al. 2002). It is possible that transfer of learning on the basis of pitch to other stimulus types, or to the same stimulus filtered into a different frequency region, is impaired by the associated differences in timbre.

The pitch specificity of $F_{0}$ discrimination learning observed in our study is independent of resolvability. In fact, learning transferred between the two stimuli with the same pitch (110 ALT and 220 SINE) despite their differences in resolvability. This result indicates that $F_{0}$ discrimination learning affects a pitch processing mechanism common to resolved and unresolved complexes. Our results, on the other hand, do not provide compelling evidence that $F_{0}$ discrimination learning affects pitch encoding mechanisms specific to resolved and unresolved complexes. Although training with a resolved complex led to greater learning for resolved complexes than did training with an unresolved complex, we did not find evidence that training with unresolved complexes leads to specific learning for unresolved complexes. Moreover, given that the 220 SINE and 246.94 SINE stimuli are only one sixth of an octave apart, the resolvability effect for resolved complexes may have been partly driven by their similarity in pitch. However, the P2 component of the EP showed the same effect of resolvability for resolved complexes manifest in the behavioral results, but it did not show effects of pitch specificity. If the P2 and behavioral changes had a common basis, this would seem to rule out the hypothesis that similarity in pitch between the 220 SINE and 246.94 SINE complexes can account for the resolvability effect for resolved complexes. However, given that P2 and behavioral changes did not significantly correlate, it is not clear that they had a common basis.

It should be kept in mind that evidence on the perceptual encoding mechanisms of different stimuli derived from learning studies is only circumstantial. Specificity of learning does not necessarily indicate differences in the encoding of two different stimuli. A psychophysical discrimination task involves several stages that include the encoding of the stimuli as well as the storage and comparison of a sensory representation of the stimuli presented in different observation intervals (Hawkey et al. 2004). Specificity of learning may reflect stimulus-specific effects at any of these levels.

This is, to the best of our knowledge, the first study to document learning for level discrimination with narrowband noise. We found no significant differences between the group trained on level discrimination and the untrained group on $F_{0}$ DL changes between the S-pre and S-post sessions. No significant differences were found also between the groups trained on $F_{0}$ discrimination and the untrained control group on level discrimination changes between the S-pre and S-post sessions. Overall, these results suggest that training on a level discrimination task with a narrowband noise does not greatly benefit $F_{0}$ discrimination and vice versa.

\section{Cortical EPs}

Two EP components were enhanced in response to harmonic complexes in listeners trained on $F_{0}$ discrimination compared to controls: the P2 and a late component from about 250 to $400 \mathrm{~ms}$. An enhancement of the P2 component, whose source has been localized in secondary auditory cortex (Shahin et al. 2003), has been found in previous studies using multiple-hour auditory training paradigms of pitch discrimination (Atienza et al. 2002; Bosnyak et al. 2004; Tong et al. 2009), voice onset time discrimination (Tremblay et al. 2001; Tremblay and Kraus 2002), and concurrent vowel segregation (Reinke et al. 2003). The lack of correlation between P2 increases and behavioral improvements after training is also consistent with previous studies (Tremblay et al. 2001, 2010; Tremblay and Kraus 2002; Sheehan et al. 2005). P2 increases have also been observed across repeated EP recording sessions in the absence of 
training (Sheehan et al. 2005; Ross and Tremblay 2009), suggesting that mere stimulus exposure may trigger the P2 amplitude increases. Tremblay et al. (2010), however, found that P2 increases over several EP sessions were larger when passive exposure during the EP session was paired with an active task at the end of the session. The results of Tremblay et al. (2010) suffer from the confound that the active task involved further, albeit short, stimulus exposure. The results of our study indicate clearly that stimulus exposure alone cannot fully explain the P2 amplitude increases observed after training. Our test for pitch learning effects on the P2 between the groups trained on $F_{0}$ discrimination and the control groups included both trained and untrained complexes. However, the contrast is still significant when only the untrained complexes are taken into account (i.e., performance improvements for complexes different to the trained ones). For these complexes, exposure to the test stimulus was the same between the groups trained on $F_{0}$ discrimination and the control groups, yet the P2 increases were larger in the former. This result indicates that active engagement on a discrimination task can lead to P2 enhancements that generalize also to untrained stimuli. It has been speculated that P2 enhancements after training reflect the consolidation of a memory trace of the stimulus (Ross and Tremblay 2009; Tremblay et al. 2010). Our finding that the P2 increases were also present for the untrained stimuli cannot be explained in terms of a memory consolidation process. Therefore, the $\mathrm{P} 2$ increases we observed must reflect, at least in part, changes in general auditory processing mechanisms.

Interestingly, the P2 enhancement after training was present for the groups trained on $F_{0}$ discrimination, but not for the group trained on level discrimination with the narrowband noise. Given that P2 training effects are often found after multiple-hour training with pitch-evoking stimuli (e.g., Atienza et al. 2002; Bosnyak et al. 2004), the present results may suggest that the training effects are specific for this type of stimulus. However, in our study, both the training task and stimuli differed between the groups trained on $F_{0}$ and level discrimination. Therefore, the differences between these two groups cannot be unequivocally ascribed to differences in the stimuli used during training. The P2 changes also partly reflected the pattern of changes in $F_{0}$ DLs; in particular, the $\mathrm{P} 2$ increase was greater for resolved complexes in listeners trained with a resolved complex compared to listeners trained with an unresolved complex. On the other hand, listeners trained with an unresolved complex did not show a greater P2 increase for unresolved complexes compared to listeners trained with resolved complexes.
The greater post-training negativity at the $\mathrm{Fz}$ electrode in the 250- to 400-ms latency window has not been previously reported in multiple-hour auditory training studies. However, increased negativities over fronto-central electrodes in similar time windows have been reported following rapid perceptual learning in auditory discrimination tasks within a single recording session (Alain et al. 2010; Ben-David et al. 2011). Alain et al. (2007) and Alain and Snyder (2008) also reported changes in an EP component around a similar time window that was more positive after rapid perceptual learning at temporal sites and inverted polarity at fronto-central sites. The morphology of this component thus appears similar to the morphology of the late EP component measured in the current study (increased post-training negativity at fronto-central sites with polarity reversal at the mastoid electrode). In the studies by Alain et al. (2007, 2010), Alain and Snyder (2008), and BenDavid et al. (2011), participants were actively attending to the stimuli and performing an auditory discrimination task during the EP recordings. The increased negativity at fronto-central sites (or increased positivity at temporal sites) was thus attributed to changes in stimulus classification or response selection processes. Since in our experiment the participants were passively listening to the stimuli while watching a movie, these interpretations of the effect are unlikely in our study. Given that the effect seemed to generalize to the untrained stimuli, it is likely that it reflects general stimulus processing changes rather than a form of stimulus memory. Further investigations are needed to fully understand the significance and functional role of these changes.

\section{Mismatch Negativity}

Winkler et al. (1997) showed that missing fundamental harmonic complexes elicit an MMN response even when concurrent spectral changes are controlled for. Our results corroborate this finding by showing that even unresolved-harmonic complexes, for which spectral information on the individual components is not available to the auditory system, can elicit an MMN response. The MMN in the present experiment was not affected by either pitch or level discrimination training. However, the MMN in the control experiment was sensitive to discriminability differences of the same magnitude as those produced by training, when that difference in discriminability was produced by changes in the stimulus. This suggests that the discriminability changes induced by training are not apparent in the neural representation reflected by the MMN. This could be because improvements in pitch discrimination after training are largely mediated by processes occurring above the level of generation of 
the MMN. However, given the non-hierarchical and parallel connectivity of the auditory pathways (Kaas and Hackett 2000), the improvement could alternatively reflect changes in processing at lower levels that do not contribute to the representation at the level of the MMN.

Our results appear to be in contrast with previous reports of MMN increases after short-term training in a task involving frequency discrimination (Atienza et al. 2002) and the larger MMN amplitude observed in musicians (Koelsch et al. 1999; Tervaniemi et al. 2001). In these studies, however, an MMN increase after training, or an MMN advantage for musicians, was present when the frequency deviant was a mistuned note presented in the context of a repeating melodic pattern (Tervaniemi et al. 2001; Atienza et al. 2002) or a mistuned partial in an otherwise harmonic chord (Koelsch et al. 1999). No MMN differences between musicians and non-musicians were observed in passive listening conditions when the frequency deviant was a pure tone presented in the context of a repeating pure tone of a different frequency (Brattico et al. 2001; Tervaniemi et al. 2005) despite the fact that musicians have lower frequency difference limens for pure tones than non-musicians (Micheyl et al. 2006). The latter presentation mode corresponds to the one employed in our study, where an $F_{0}$ deviant harmonic complex is presented in the context of a repeating harmonic complex with a different $F_{0}$. Our results, therefore, in line with those of Tervaniemi et al. (2005), suggest that the fine pitch discrimination skills acquired with training are not necessarily reflected in better pre-attentive detection of pitch deviance per se. The MMN instead seems to reflect training-related improvements in the detection of deviant melodic or harmonic patterns.

A possible concern with our results comes from the use of the identity MMN paradigm. This paradigm has the merit of controlling for acoustical differences between the deviant and standard sounds. However, since the "standard" used is a continuous repetition of the same stimulus that was used as a "deviant" in the oddball paradigm, it may exacerbate the effects of adaptation on the MMN. A reanalysis of our data using the traditional MMN derivation, however, did not reveal any qualitative differences with the results obtained using the identity MMN derivation.

Given that we recorded the baseline MMN after the first behavioral session, it is possible that we missed potential MMN changes caused by fast perceptual learning. In a recent study, Tong et al. (2009) found an MMN enhancement after a single session of pure tone frequency discrimination training. Their baseline MMN recordings, however, were obtained before pitch discrimination accuracy was measured behaviorally and may thus reflect fast perceptual learning occurring during the first behavioral assessment session. We chose to run the baseline EP recording session after the first behavioral assessment session because this allowed us to set the $F_{0}$ difference between the standard and the deviant complex in the EP recordings at the participant's $F_{0}$ discrimination threshold and because we were mostly interested in the slow $F_{0}$ discrimination learning that continues over multiple sessions (Grimault et al. 2002; Demany and Semal 2002; Carcagno and Plack 2011) rather than the fast perceptual learning that can occur even in the course of a single session.

\section{CONCLUSIONS}

The results of our study indicate that pitch discrimination training leads to improvements in $F_{0}$ discrimination that are partly pitch-specific. These improvements are paralleled by changes of sensoryevoked potentials, the $\mathrm{P} 2$, and a later component in the 250- to 400-ms time window, which appear to generalize to untrained stimuli. The changes in sensory processing indexed by the $\mathrm{P} 2$ increase do not seem to automatically sharpen the pre-attentive detection of pitch deviants as indexed by the MMN.

\section{ACKNOWLEDGMENTS}

We would like to thank R. P. Carlyon, K. Mattock, M. Turgeon, and two anonymous reviewers for constructive comments. S. Carcagno was supported in part by an EPSRC doctoral training award.

\section{Conflict of interest disclosure statement}

I declare, on behalf of all authors, that we have no conflicts of interest with the subject matter or materials discussed in this manuscript.

Samuele Carcagno

\section{REFERENCES}

Alain C, Snyder JS, He Y, Reinke KS (2007) Changes in auditory cortex parallel rapid perceptual learning. Cereb Cortex 17:1074-1084

Alain C, SNYDER JS (2008) Age-related differences in auditory evoked responses during rapid perceptual learning. Clin Neurophysiol 119:356-366

Alain C, Campeanu S, Tremblay K (2010) Changes in sensory evoked responses coincide with rapid improvement in speech identification performance. J Cogn Neurosci 22:392-403

Atienza M, Cantero JL, Dominguez-Marin E (2002) The time course of neural changes underlying auditory perceptual learning. Learn Mem 9:138-150 
Ben-David BM, Campeanu S, Tremblay KL, Alain C (2011) Auditory evoked potentials dissociate rapid perceptual learning from task repetition without learning. Psychophysiology. doi:10.1111/ j.1469-8986.2010.01139.x

Bernstein JG, Oxenham AJ (2003) Pitch discrimination of diotic and dichotic tone complexes: harmonic resolvability or harmonic number? J Acoust Soc Am 113:3323-3334

BosNyak DJ, Eaton RA, Roberts LE (2004) Distributed auditory cortical representations are modified when non-musicians are trained at pitch discrimination with $40 \mathrm{~Hz}$ amplitude modulated tones. Cereb Cortex 14:1088-1099

Brattico E, Näätänen R, Tervaniemi M (2001) Context effects on pitch perception in musicians and nonmusicians: evidence from event-related-potential recordings. Music Percept 19:199-222

Buus S, Florentine M (1991) Psychometric functions for level discrimination. J Acoust Soc Am 90:1371-1380

Carcagno S, Plack CJ (2011) Subcortical plasticity following perceptual learning in a pitch discrimination task. J Assoc Res Otolaryngol 12:89-100

Carlyon RP, Shackleton TM (1994) Comparing the fundamental frequencies of resolved and unresolved harmonics: evidence for two pitch mechanisms? J Acoust Soc Am 95:3541-3554

Chandrasekaran B, Krishnan A, Gandour JT (2007) Mismatch negativity to pitch contours is influenced by language experience. Brain Res 1128:148-156

de Cheveigné A (2005) Pitch perception models. In: Plack CJ, Oxenham AJ (eds) Pitch: neural coding and perception. Springer, New York

Conover W, Johnson M, Johnson M (1981) A comparative study of tests for homogeneity of variances, with applications to the outer continental shelf bidding data. Technometrics 23:351361

Demany L, Semal C (2002) Learning to perceive pitch differences. J Acoust Soc Am 111:1377-1388

Fitzgerald MB, Wright BA (2005) A perceptual learning investigation of the pitch elicited by amplitude-modulated noise. J Acoust Soc Am 118:3794-3803

Glasberg BR, Moore BCJ (1990) Derivation of auditory filter shapes from notched-noise data. Hear Res 47:103-138

Grimault N, Micheyl C, Carlyon RP, Bacon SP, Collet L (2003) Learning in discrimination of frequency or modulation rate: generalization to fundamental frequency discrimination. Hear Res 184:41-50

Grimault N, Micheyl C, Cariyon RP, Collet L (2002) Evidence for two pitch encoding mechanisms using a selective auditory training paradigm. Percept Psychophys 64:189-197

Hawkey DJC, Amitay S, Moore DR (2004) Early and rapid perceptual learning. Nat Neurosci 7:1055-1056

Houtsma AJM, Smurzynski J (1990) Pitch identification and discrimination for complex tones with many harmonics. J Acoust Soc Am 87:304-310

KaAs JH, Hackett TA (2000) Subdivisions of auditory cortex and processing streams in primates. Proc Natl Acad Sci USA 97:11793-11799

Koelsch S, Schröger E, Tervaniemi M (1999) Superior pre-attentive auditory processing in musicians. Neuroreport 10:1309-1313

LevitT H (1971) Transformed up-down methods in psychoacoustics. J Acoust Soc Am 49:467-477
LICKLIDER JCR (1956) Auditory frequency analysis. In: Cherry C (ed) Information theory. Academic, New York, pp 253-268

Micheyl C, Delhommeau K, Perrot X, Oxenham AJ (2006) Influence of musical and psychoacoustical training on pitch discrimination. Hear Res 219:36-47

Novitski N, Tervaniemi M, Huotilainen M, NÄÄtänen R (2004) Frequency discrimination at different frequency levels as indexed by electrophysiological and behavioral measures. Brain Res Cogn Brain Res 20:26-36

Plack CJ, Oxenham AJ (2005) The psychophysics of pitch. In: Plack CJ, Oxenham AJ (eds) Pitch: neural coding and perception. Springer, New York

Plack CJ, Carlyon RP (1995) Differences in frequency modulation detection and fundamental frequency discrimination between complex tones consisting of resolved and unresolved harmonics. J Acoust Soc Am 98:1355-1364

Reinke KS, He Y, Wang C, Alain C (2003) Perceptual learning modulates sensory evoked response during vowel segregation. Brain Res Cogn Brain Res 17:781-791

Ross B, Tremblay K (2009) Stimulus experience modifies auditory neuromagnetic responses in young and older listeners. Hear Res 248:48-59

SATTERTHWAite F (1946) An approximate distribution of estimates of variance components. Biometrics Bull 2:110-114

Shackleton TM, Carlyon RP (1994) The role of resolved and unresolved harmonics in pitch perception and frequency modulation discrimination. J Acoust Soc Am 95:3529-3540

Shahin A, Bosnyak DJ, Trainor LJ, Roberts LE (2003) Enhancement of neuroplastic P2 and N1c auditory evoked potentials in musicians. J Neurosci 23:5545-5552

SheEhAn KA, McArthur GM, Bishop DVM (2005) Is discrimination training necessary to cause changes in the $\mathrm{P} 2$ auditory eventrelated brain potential to speech sounds? Brain Res Cogn Brain Res 25:547-553

Tervaniemi M, Just V, Koelsch S, Widmann A, Schröger E (2005) Pitch discrimination accuracy in musicians vs nonmusicians: an eventrelated potential and behavioral study. Exp Brain Res 161:1-10

Tervaniemi M, Rytkönen M, Schröger E, Ilmoniemi RJ, NäÄtänen R (2001) Superior formation of cortical memory traces for melodic patterns in musicians. Learn Mem 8:295-300

Tong Y, Melara RD, Rao A (2009) P2 enhancement from auditory discrimination training is associated with improved reaction times. Brain Res 1297:80-88

Tremblay K, Kraus N, McGee T, Ponton C, Otis B (2001) Central auditory plasticity: changes in the N1-P2 complex after speechsound training. Ear Hear 22:79-90

Tremblay KL, Inoue K, McClannahan K, Ross B (2010) Repeated stimulus exposure alters the way sound is encoded in the human brain. PLOS ONE 5:e10283

Tremblay KL, Kraus N (2002) Auditory training induces asymmetrical changes in cortical neural activity. J Speech Lang Hear Res 45:564-572

Winkler I, Tervaniemi M, NÄÄTÄnen R (1997) Two separate codes for missing-fundamental pitch in the human auditory cortex. J Acoust Soc Am 102:1072-1082

Wright BA, Zhang Y (2009) A review of the generalization of auditory learning. Philos Trans R Soc Lond B Biol Sci 364:301311 